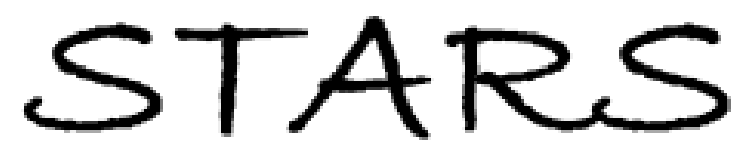

University of Central Florida

STARS

$1-1-2013$

\title{
Millimeter-wave photoresponse due to excitation of two- dimensional plasmons in InGaAs/InP high-electron-mobility transistors
}

N. Nader Esfahani

University of Central Florida

R. E. Peale

University of Central Florida

W. R. Buchwald

C. J. Fredricksen

University of Central Florida

J. R. Hendrickson

Find similar works at: https://stars.library.ucf.edu/facultybib2010

University of Central Florida Libraries http://library.ucf.edu See next page for additional authors

This Article is brought to you for free and open access by the Faculty Bibliography at STARS. It has been accepted for inclusion in Faculty Bibliography 2010s by an authorized administrator of STARS. For more information, please contact STARS@ucf.edu.

\section{Recommended Citation}

Esfahani, N. Nader; Peale, R. E.; Buchwald, W. R.; Fredricksen, C. J.; Hendrickson, J. R.; and Cleary, J. W., "Millimeter-wave photoresponse due to excitation of two-dimensional plasmons in InGaAs/InP highelectron-mobility transistors" (2013). Faculty Bibliography 2010s. 3954.

https://stars.library.ucf.edu/facultybib2010/3954

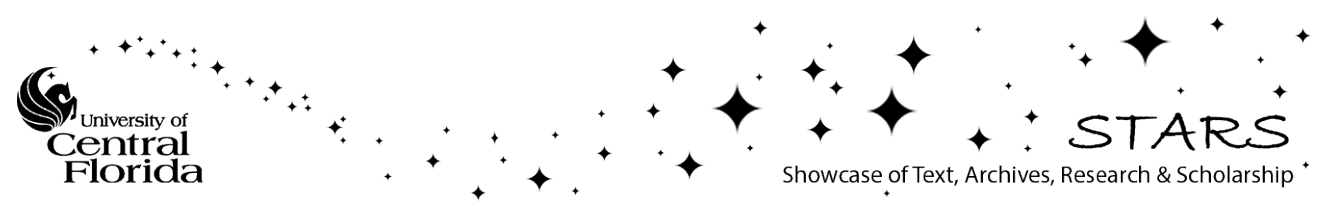


Authors

N. Nader Esfahani, R. E. Peale, W. R. Buchwald, C. J. Fredricksen, J. R. Hendrickson, and J. W. Cleary 


\section{Millimeter-wave photoresponse due to excitation of two-dimensional plasmons in InGaAs/InP high-electron-mobility transistors}

Cite as: J. Appl. Phys. 114, 033105 (2013); https://doi.org/10.1063/1.4813511

Submitted: 17 June 2013 . Accepted: 23 June 2013 . Published Online: 17 July 2013

N. Nader Esfahani, R. E. Peale, W. R. Buchwald, C. J. Fredricksen, J. R. Hendrickson, and J. W. Cleary
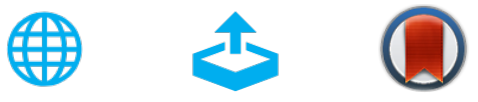

View Online

Export Citation

\section{ARTICLES YOU MAY BE INTERESTED IN}

Versatile silicon-waveguide supercontinuum for coherent mid-infrared spectroscopy APL Photonics 3, 036102 (2018); https://doi.org/10.1063/1.5006914

Design, fabrication, and metrology of $10 \times 100$ multi-planar integrated photonic routing manifolds for neural networks

APL Photonics 3, 106101 (2018); https://doi.org/10.1063/1.5039641

Metasurface optical antireflection coating

Applied Physics Letters 105, 241113 (2014); https://doi.org/10.1063/1.4904827

\section{Applied Physics Reviews} Now accepting original research

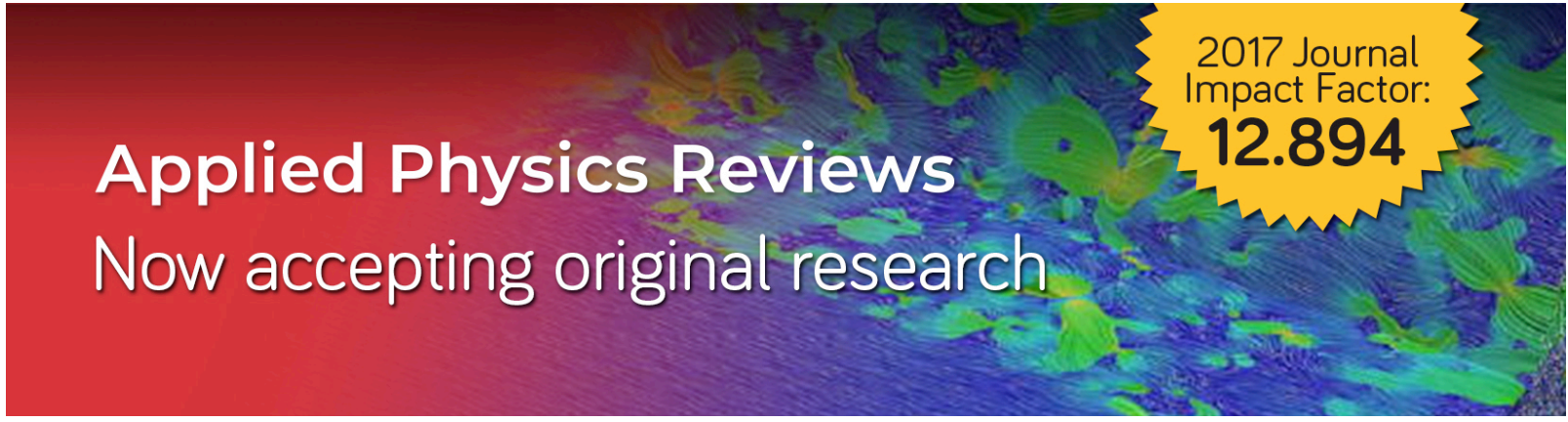




\title{
Millimeter-wave photoresponse due to excitation of two-dimensional plasmons in InGaAs/InP high-electron-mobility transistors
}

\author{
N. Nader Esfahani, ${ }^{1,2,3, a)}$ R. E. Peale, ${ }^{2}$ W. R. Buchwald, ${ }^{1,4}$ C. J. Fredricksen, ${ }^{2}$ \\ J. R. Hendrickson, ${ }^{3}$ and J. W. Cleary ${ }^{3}$ \\ ${ }^{1}$ Solid State Scientific Corporation, 12 Simon St., Nashua, New Hampshire 03060, USA \\ ${ }^{2}$ Department of Physics, University of Central Florida, Orlando, Florida 32816, USA \\ ${ }^{3}$ Air Force Research Laboratory, Sensors Directorate, 2241 Avionics Circle, Wright Patterson AFB, \\ Ohio 45433, USA \\ ${ }^{4}$ Department of Physics, University of Massachusetts, Boston, Massachusetts 02125, USA
}

(Received 17 June 2013; accepted 23 June 2013; published online 17 July 2013)

A polarized photoresponse to mm-wave radiation over the frequency range of 40 to $108 \mathrm{GHz}$ is demonstrated in a grating-gated high electron mobility transistor (HEMT) formed by an InGaAs/InP heterostructure. The photoresponse is observed within the plasmon resonance absorption band of the HEMT, whose gate consists of a $9 \mu \mathrm{m}$ period grating that couples incident radiation to plasmons in the 2D electron gas. Gate-bias changes the channel carrier concentration, causing a corresponding change in photoresponse in agreement with theoretical expectations for the shift in the plasmon resonance band. The noise equivalent power is estimated to be $235 \mathrm{pW} / \mathrm{Hz}^{1 / 2}$. C 2013 AIP Publishing LLC. [http://dx.doi.org/10.1063/1.4813511]

\section{INTRODUCTION}

Plasmonic grating-gated high electron mobility transistors (HEMTs) have potential as fast, chip-scale and frequencytunable $\mathrm{THz}$ detectors. The tunable resonant absorption of plasmons in the 2D electron gas (2DEG) of grating-gated MOSFETs and HEMTs has been reported for a number of material systems which include Si-inversion layers, ${ }^{1}$ GaAs field effect transistors, ${ }^{2-7}$ InGaAs/InP HEMTs, ${ }^{8}$ and GaN HEMTs. ${ }^{9}$ A coupling structure, such as grating ${ }^{1-4,8,9}$ or antenna, ${ }^{6,7}$ is required to match momentum between free space radiation and 2D-plasmons, whose resonance frequency depends on the 2DEG sheet charge density, which in turn depends linearly on the external gate-voltage. ${ }^{8}$ This provides a means to not only excite plasmon modes but also to actively tune the absorption resonance.

For detector applications, the necessary transduction of this absorption to a measurable change in channel conductance has been so far demonstrated only in GaAs-based devices. ${ }^{2-7}$ In this material system, the coupling structure has been evolved from a uniform metallized grating ${ }^{2-5}$ to structures which use split-gratings 5 or which integrate a metallic grating, a bolometric detection method, and a log-periodic antenna $^{6,7}$ to increase responsivity and reduce the noise-equivalent-power (NEP) of the detector.

This paper presents the observation of such transduction for HEMTs fabricated in the InP materials system. Specifically, we report gate-bias-tunable electrical photoresponse in InGaAs/InP HEMTs to mm-wave radiation at 40-60 and $83-108 \mathrm{GHz}$ at $4 \mathrm{~K}$ device temperature. Preliminary results and device background characterization were presented in Refs. 10 and 11. This frequency range was chosen due to the availability of table-top mm-range backward wave oscillators (BWOs) with high intensity stability and frequency

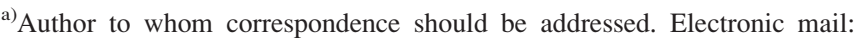
Nima.Nader.ctr@wpafb.af.mil
}

agility. Though $\mathrm{THz}$ tunable plasmon resonances were observed in similar material with $0.5 \mu \mathrm{m}$ gratings, ${ }^{8}$ our attempt to observe resonant photoresponse in that device using a free electron laser was unsuccessful due to experimental intensity fluctuations and a strong non-linear non-resonant photo-response. ${ }^{16}$ This motivated the change in our grating period to $9 \mu \mathrm{m}$ to allow plasmon excitation by ultrastable BWOs at mm-wavelengths, which has enabled us to observe the desired effect. We compare the results for this device with $\mathrm{mm}$-wave/THz detectors discussed in Ref. 6. The long-term objective is to scale the device so that it operates as a tunable resonant detector at $\mathrm{THz}$ frequencies by utilizing a grating with a smaller, sub-micron period. Such a device can have applications as a chip-scale, frequency tunable detector in high-speed InP-based electro-optic integrated circuits.

\section{EXPERIMENTAL DETAILS}

The devices of this work were fabricated from a molecular-beam-epitaxial grown InGaAs/InP HEMT structure with two $\delta$-doped layers (Figure 1). The $\delta$-doping, instead of a thick uniformly doped layer, was chosen to avoid anomalous free-carrier effects. ${ }^{12,13}$ Device fabrication consisted of first etching the active layers into mesas for electrical isolation. This was followed by the patterning and deposition of electron-beam evaporated $\mathrm{Ti} / \mathrm{Au}(500 \mathrm{~nm} / 2500 \mathrm{~nm})$ metal pads, subsequently rapid thermal annealed to form ohmic source and drain contacts. Gates were formed by first removing the doped-InGaAs cap layer that facilitated source and drain ohmic contact formation. A $20 \mathrm{~nm}$ layer of $\mathrm{Ti}$ was then deposited on the $195 \mu \mathrm{m} \times 250 \mu \mathrm{m}$ gate region to act as a semitransparent gate metal to allow uniform gate-bias control of the 2DEG sheet charge concentration. The grating, with period $a=9 \mu \mathrm{m}$ and openings $t=0.22 a$ wide, was patterned by optical lithography over the semi-transparent gate. The grating bars consisted of Ti/Au $(15 \mathrm{~nm} / 100 \mathrm{~nm})$. Source, gate, and drain bond pads were fabricated by photolithography 




FIG. 1. Schematic of the grating-gated InP-based HEMT. The epitaxially grown materials are indicated as $\mathrm{A}=\mathrm{In}_{0.48} \mathrm{Al}_{0.52} \mathrm{As}$ with $\delta$-doping at the interface with $\mathrm{B}, \mathrm{B}=$ undoped $\mathrm{In}_{0.48} \mathrm{Al}_{0.52} \mathrm{As}$ spacer, $\mathrm{C}=\mathrm{In}_{0.32} \mathrm{Ga}_{0.68} \mathrm{As}$ channel, and $\mathrm{E}=\mathrm{In}_{0.48} \mathrm{Al}_{0.52} \mathrm{As}$ buffer layer. Radiation is incident on the grating-gate from the left. The black curve, calculated by finite element method (Silvaco Atlas), indicates the charge density distribution.

using Ti/Au $(50 \mathrm{~nm} / 250 \mathrm{~nm})$. The sample was mounted in a TO-5 transistor package. An optical microscope image of the device is presented in Figure 2.

The device was fixed to a copper cold finger inside a Janis SHI-4 closed cycle cryostat. A silicon diode temperature sensor, thermally contacted to the cold finger below the device, was connected to a temperature controller to maintain a $4 \mathrm{~K}$ temperature. Sheet charge density $n_{s}$ and relaxation time $\tau$ were determined from $4 \mathrm{~K}$ Source-Drain I-V curves according to Ref. 14. This analysis has been archived in Ref. 11. Relaxation time $\tau$ at $4 \mathrm{~K}$ was determined to be $0.37 \mathrm{ps}$ and sheet charge densities $n_{s}$ to be $(1.39,1.21$, and 1.04$) \times 10^{12} \mathrm{~cm}^{-2}$ at gate biases of $(0$, -0.1 , and -0.2$) \mathrm{V}$, respectively.

Figure 3 presents a schematic of the mm-wave experiment. A $100 \mathrm{~Hz}$ sine wave with adjustable DC offset modulated and swept the output frequency of a Siemens BWO. The modulation amplitude was $100 \mathrm{MHz}$ or $\sim 0.1 \%$ of the central frequency. The same control signal was high-pass filtered and amplified for the lock-in reference. A crystal detector connected via a $10 \mathrm{~dB}$ directional coupler monitored the mm-wave power, and this signal was used for power leveling the BWO. The power measured with a crystal detector in place of the horn was $2.6 \mathrm{~mW}$ $(1.7 \mathrm{~mW})$ for the low (high) frequency range.

The mm-waves from the BWO are conducted to the cryostat with rectangular waveguides and a polarizationpreserving horn. The radiation passed through a polyethylene

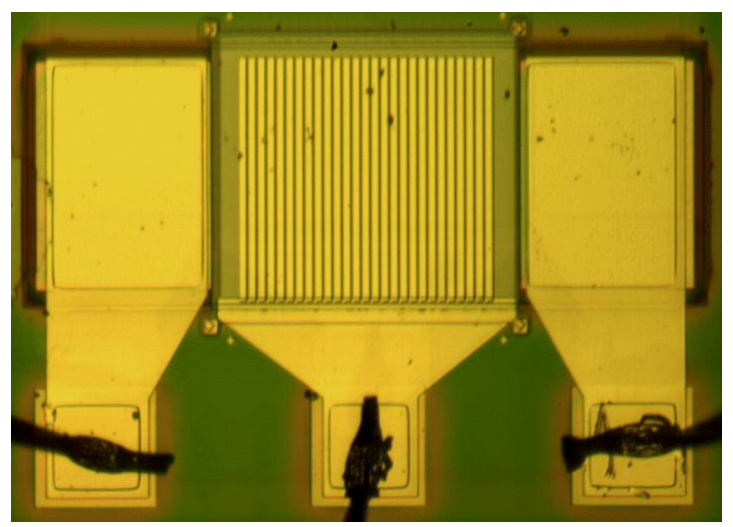

FIG. 2. Optical microscope image of the grating gated InP-based HEMT.

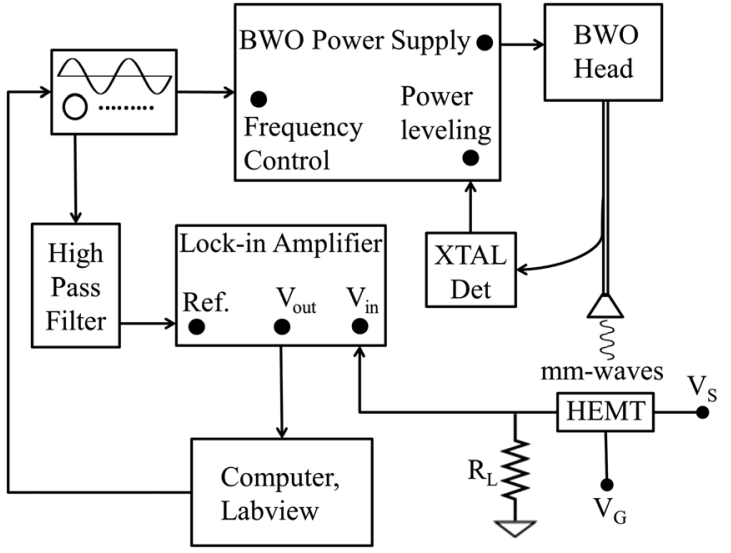

FIG. 3. Schematic of the experimental setup.

window into the vacuum of the cryostat to irradiate the device. The BWO head could be rotated about the optical axis to study polarization effects. During measurements, a constant $0.5 \mathrm{~V}$ was applied to the source while the gate was biased at $0,-0.1$, and $-0.2 \mathrm{~V}$, all with respect to ground, with the drain connected to ground via a $100 \Omega$ load.

\section{THEORETICAL CONSIDERATIONS}

The dispersion relation for 2D-plasmons in a device with a metalized grating is ${ }^{1}$

$$
\omega_{n}^{2}=\frac{e^{2} n_{s} q_{n}}{m^{*} \varepsilon_{0}}\left[\varepsilon_{b}+\varepsilon_{t} \operatorname{coth}\left(q_{n} d\right)\right]^{-1},
$$

where $\omega_{n}$ is the frequency of the $\mathrm{n}^{\text {th }}$ integer-order plasmon, $e$ is the electron charge, $m^{*}$ is the effective mass, $d$ is the 2DEG depth from the grating (Fig. 1), $\varepsilon_{0}$ is the permittivity of free space, and $\varepsilon_{t}\left(\varepsilon_{b}\right)$ are the relative permittivity of the semiconductor layers above (beneath) the $2 \mathrm{DEG}$. The wavevector of excited plasmons $q_{n}$ takes discrete values $2 \pi n / a$.

Source-drain current $I_{S D}$ is recorded using a lock-in amplifier synchronized with the $\mathrm{mm}$-wave frequency modulation $\Delta f$. The lock-in input is the voltage drop $V_{L}$ across the load $R_{L}$ and the output is given by ${ }^{11}$

$$
V_{\text {out }}\left(V_{g}, f\right)=\frac{2}{\pi}\left(\frac{d I_{S D}\left(V_{g}, f\right)}{d f}\right) R_{L} \Delta f .
$$

The current change $d I_{S D}$ may be written as $d(B W A)$, where $B$ is a coupling factor between plasmon absorption and channel conductance, $W$ is the incident radiation power, and $A$ is the plasmon absorptance. Assuming $B$ to be frequency independent, and with power-leveling, we have

$$
V_{\text {out }}\left(V_{g}, f\right)=\frac{2}{\pi} B W\left(\frac{d A\left(V_{g}, f\right)}{d f}\right) R_{L} \Delta f .
$$

Assuming constant front surface reflectance $R$, we obtain absorptance from $A=1-R-T$, where the transmittance spectra $T$ for different gate biases are calculated as in Refs. 8, 13, and 15.

\section{RESULTS}

Calculated plasmon resonance spectra are presented in Figure 4 for three different grating periods $(0.5,2$, and $9 \mu \mathrm{m})$, 




FIG. 4. Calculated absorptance spectra for three different grating periods of 9,2 , and $0.5 \mu \mathrm{m}$. Fundamental and harmonics are labeled in each spectrum.

while all other parameters apply to our experimental device. In Fig. 4, we see that increasing the grating period from 0.5 to 2 to $9 \mu \mathrm{m}$ shifts the plasmon fundamental from 2.82 to 0.85 to $0.36 \mathrm{THz}$, respectively, in agreement with Eq. (1). Fig. 4 also shows that as the grating period is increased, the harmonics move closer together. For the grating period of $9 \mu \mathrm{m}$, which puts the fundamental in range of our BWOs, the harmonics have merged with the fundamental. This causes the plasmon absorption band to be quite broad, with a full-width-half-maximum (FWHM) of $\sim 700 \mathrm{GHz}$.

Figure 5(a) presents calculated absorption spectra for the device of this work at $4 \mathrm{~K}$ for frequencies up to $400 \mathrm{GHz}$ at gate-biases of $0,-0.1$, and $-0.2 \mathrm{~V}$. The figure includes only the low frequency portion of the broad plasmon resonance bands, whose peaks occur at 365,340 , and $320 \mathrm{GHz}$ as indicated by triangle symbols above the respective curves. The redshift with increasing negative $V_{g}$, and thus decreasing $n_{s}$, is given by Eq. (1). The absorption is also seen to become shallower as expected due to the reduction of the plasmonsupporting charge carrier concentration. The BWO ranges

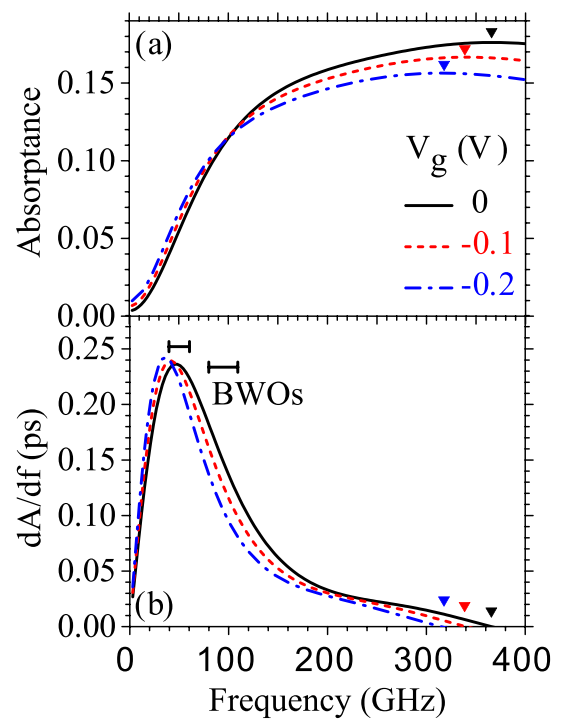

FIG. 5. (a) Calculated absorptance spectra. Absorptance maxima are marked by symbols. (b) Calculated $d A / d f$ spectra. The horizontal bars indicate the available BWO ranges.
(Fig. 5(b)) lie at frequencies where the absorptance curves are $35 \%$ and $65 \%$ of the maximum value, so that our experimental wavelengths are within the plasmon resonance absorption bandwidth.

From Eq. (3), the lock-in output is proportional to $d A / d f$, whose spectrum is presented in Figure 5(b) with the ranges of the available BWOs indicated. Maxima of plasmon absorption bands are marked by triangle symbols, where the derivatives are zero. From these curves, we expect the response to decrease with increasing frequency.

The measured photoresponse of the device is shown in Figure 6 for gate-biases of $0,-0.1$, and $-0.2 \mathrm{~V}$ as black, red, and blue curves, respectively. The effect was measured over the full $40-60 \mathrm{GHz}$ and $83-108 \mathrm{GHz}$ ranges, but only a portion of each measured spectrum, and only the nominal perpendicular polarization data (inset schematic), is presented. The detected photoresponse shows sharp features at discrete frequencies as opposed to the continuous electrical response expected from Fig. 5 and Eq. (3). This is explained by interference and the formation of standing waves, which is a well-known experimental artifact in mm-wave spectroscopy. When standing wave nodes occur at the grating no plasmons can be excited, and hence there can be no electrical response. At frequencies where anti-nodes occur, excitation of plasmons and a corresponding electrical response may occur. Although the known standing wave phenomenon somewhat obscures the results, it is clear each individual peak weakens with increasing negative gate bias suggesting reduced plasmon interactions with the decreasing 2DEG sheet charge as expected. The noise of the system was measured by blocking the radiation and recording the lock-in

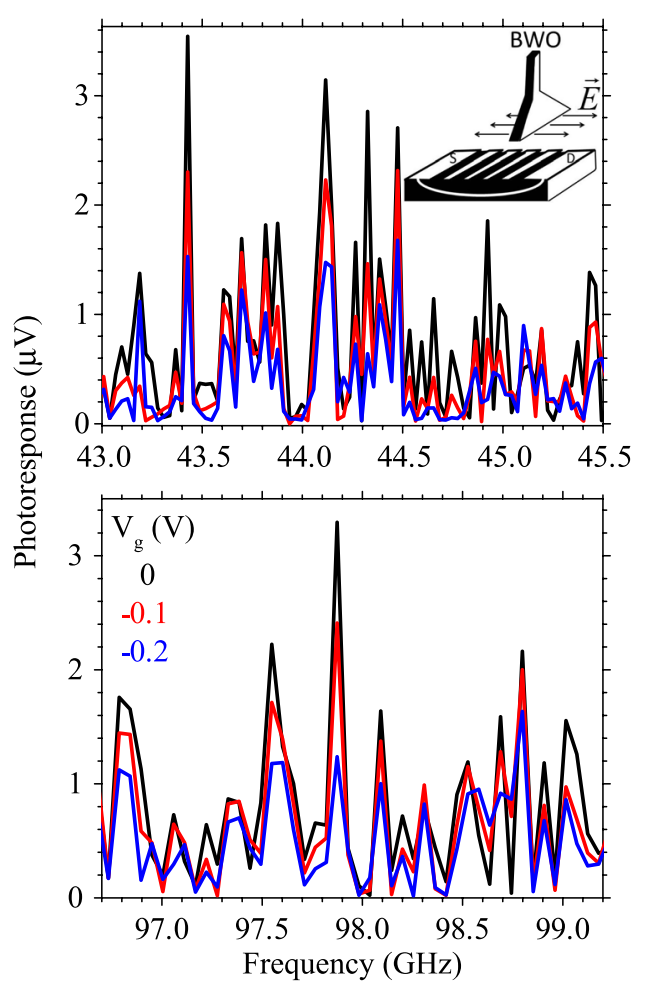

FIG. 6. Photoresponse of the HEMT at $\mathrm{T}=4 \mathrm{~K}$ to $\mathrm{mm}$-wave radiation for three gate biases. Data are for nominal perpendicular polarization as expected from the horn orientation (inset schematic). 
output, and its root-mean-square (RMS) value was determined to be $\sim 45 \mathrm{nV}$.

Although the response for parallel polarization is expected to be zero, since it should fail to polarize the grating and produce plasmon-exciting local fields, a similar but weaker response was in fact observed for this nominal polarization. Using two gain horns and a wire-grid polarizer, the radiation output by the horn was confirmed to be highly polarized. However, when the polyethylene cryostat window was placed between the crossed horns, substantial collected signal appears. This is attributed to rotation of the polarization due to refraction and scattering from imperfections in or on the window. A tangle of wire with random orientations produces an even stronger response, showing that reflections inside the cryostat can also scramble the polarization. This explains the unexpected photoresponse for nominally parallel polarization. This polarization study is archived in Ref. 11. These studies simply confirm that the well-known possible change of polarization angle on reflection or refraction ${ }^{17}$ is likely occurring in our experiment.

Linear fits to the data in each full frequency range represent the average photoresponse and frequency dependence for each $V_{g}$. Figure 7 compares such fits to the theoretical $d A / d f$ spectra from Figure 5(b). To focus purely on the frequency dependence, each fit is vertically scaled by different values to match the corresponding theoretical $d A / d f$ curves. In the low frequency range, experiment is matched to theory at $47 \mathrm{GHz}$, while in the high frequency range, this occurs at 96.4 GHz. The scaling factors increase monotonically with increasing $V_{g}$ magnitude by less than $50 \%$ for the low frequency range. In the high frequency range, these factors differ by less than $2 \%$, which is within the experimental uncertainty. In neither case is the vertical ordering of the fits changed by the scaling. The need for different scaling factors in the low frequency range implies that the coupling coefficient $B$ depends on gate bias. The coupling between $\mathrm{mm}-$ waves and plasmons should be completely accounted for by the theory represented by Fig. 5, but the coupling between plasmons and transport lacks a theory, and this latter coupling may depend on $n_{s}$, in principle.

Linear fits shift downward with increasingly negative gate bias for all of the high frequency range and for all but

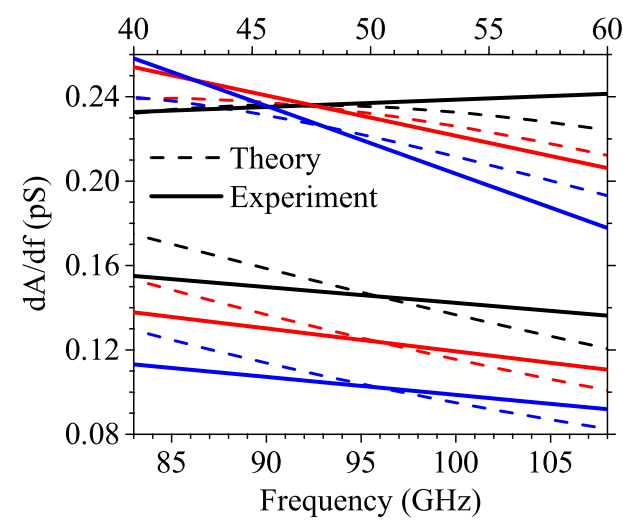

FIG. 7. Comparison of theoretical $d A / d f$ curves with linear fits to measured photoresponse data for nominal perpendicular polarization. The upper (lower) group of curves corresponds to the upper (lower) frequency axis. the $V_{g}=0$ case for the low frequency range, in qualitative agreement with the theory. Interestingly, the ordering of the linear fits at $40 \mathrm{GHz}$ is opposite that at $60 \mathrm{GHz}$, just as occurs with the $d A / d f$ curves. Linear fits obviously cannot reflect the curvature in the $d A / d f$ spectra in this low frequency range. In the high frequency region, the $d A / d f$ spectra are more nearly linear. That the fit slopes differ from theory can be attributed to several factors, namely, standing waves, monotonic decrease in power with increasing wavelength due to waveguide attenuation, and monotonic decrease in power with decreasing wavelength due to scattering. It is also interesting that the magnitude of the high frequency response is approximately half that for the low frequency range, though this apparent agreement with Fig. 5(b) is partly coincidental since the high frequency BWO outputs only $65 \%$ as much power as the low frequency one. The agreement between experiment and theory in both frequency and gate-bias dependence, though not exact, is sufficient to support the interpretation that the photoresponse is due to absorption by plasmons within their resonance band.

\section{DISCUSSION AND SUMMARY}

Further calculations, like those in Fig. 4 but for non-zero applied gate bias, show that the shift with gate bias of the fundamental decreases strongly with increasing gate period. While a gate bias of $-0.1 \mathrm{~V}$ red shifts the $2.82 \mathrm{THz}$ fundamental for the $0.5-\mu \mathrm{m}$-period grating by $200 \mathrm{GHz}$ (half the resonance line width), the shift for the $9 \mu \mathrm{m}$ period is only $\sim 20 \mathrm{GHz}$ (only $3 \%$ of the resonance band width). Thus, the detector designed for mm-waves has nearly lost many of the attractive features that make tunable 2D plasmonic HEMT detectors potentially so interesting at $\mathrm{THz}$ frequencies. However, the superior qualities of mm-wave BWOs as an excitation source strongly motivated our decision to seek a plasmonic photoresponse first at the longer wavelengths, and this has evidently proven successful.

The peak response in Fig. 6 is $5 \mu \mathrm{V}$ for the $83-108 \mathrm{GHz}$ frequency range, but there were a few points in the low frequency range which had up to $10 \mu \mathrm{V}$ peak response. These correspond to a mm-wave induced change in the current through the device of $0.1 \mu \mathrm{A}$. The channel resistance at $4 \mathrm{~K}$ was measured to be $700 \Omega$. Using the voltage divider formula, we find that the change in the channel resistance due to absorption of mm-waves by plasmons is $0.13 \Omega$ or $0.02 \%$. This value exceeds by an order of magnitude the value we determine $(0.01 \Omega)$ from the data presented for AlGaAs/ GaAs HEMTs at $763 \mathrm{GHz}$ in Ref. 2.

We may estimate the responsivity (for our specific circuit configuration) as follows. Assuming the $2.6 \mathrm{~mW} \mathrm{~mm}$-wave power at the input to the horn is uniformly distributed over its $300 \mathrm{~mm}^{2}$ aperture, the intensity leaving the horn is $9 \mu \mathrm{W} / \mathrm{mm}^{2}$. After diverging on its several $\mathrm{cm}$ path to the device, with attenuation and scattering by the cryostat window, the intensity may be reduced by at least $10 \times$ at the sample. The total power incident on the $195 \mu \mathrm{m} \times 250 \mu \mathrm{m}$ gate is then about $42 \mathrm{nW}$. This gives a responsivity estimate of $240 \mathrm{~V} / \mathrm{W}$. We may also estimate the NEP of the device as a detector. With maximum signal-tonoise ratio (SNR) of about 220, $42 \mathrm{nW}$ mm-wave power (P) 
incident on the gate, and lock-in time constant of $3 \mathrm{~s}$ giving the bandwidth $\Delta f$ of $0.66 \mathrm{~Hz}$, we estimate $N E P=P /\left(S N R \times \Delta f^{1 / 2}\right)$ $=235 \mathrm{pW} / \mathrm{Hz}^{1 / 2}$.

Next, we compare the performance characteristics with other 2D-plasmonic detectors. According to Ref. 6, responsivity and NEP values for GaAs-based HEMT detectors of $140 \mathrm{GHz}$ waves are $600 \mathrm{~V} / \mathrm{W}$ and $10 \mathrm{nW} / \mathrm{Hz}^{1 / 2}$, respectively. Evidently, we have achieved a factor of 40 better $\mathrm{NEP}$ in the InP/InGaAs device, though it is 2.5 times less responsive.

In conclusion, the electrical photoresponse to mm-wave radiation has been observed in InGaAs/InP HEMTs at frequencies well within the plasmon resonance bandwidth. We attribute photoresponse to excitation of plasmons in the 2DEG and transduction of that absorption to a change in channel conductance, because results agree with theoretical predictions in a number of significant ways. Parameters such as responsivity and noise equivalent power were estimated and compared to similar devices fabricated in the GaAs materials system. Such a device may find application as a chip-scale tunable mm-wave and sub-THz detector. However, the narrower bandwidth and broader tuning of the plasmonic response when the device is scaled with smaller grating period to $\mathrm{THz}$ frequencies would be more attractive.

\section{ACKNOWLEDGMENTS}

This work was supported by Air Force Office of Scientific Research. N.N.E. and J.W.C. acknowledge AFOSR LRIR No. 12RY10COR (Program Officer Dr. Gernot Pomrenke). R.E.P. acknowledges Grant No. FA95501010030. J.R.H. would also like to acknowledge support from AFOSR under LRIR No. 12RY05COR.

${ }^{1}$ S. J. Allen, D. C. Tsui, and R. A. Logan, Phys. Rev. Lett. 38, 980 (1977).

${ }^{2}$ E. A. Shaner, M. Lee, M. C. Wanke, A. D. Grine, J. L. Reno, and S. J. Allen, Appl. Phys. Lett. 87, 193507 (2005).

${ }^{3}$ X. G. Peralta, S. J. Allen, M. C. Wanke, N. E. Harff, J. A. Simmon, M. P. Lilly, J. L. Reno, P. J. Burke, and J. P. Eisenstein, Appl. Phys. Lett. 81, 1627 (2002).

${ }^{4}$ W. Knap, Y. Deng, S. Rumyantsev, and M. S. Shur, Appl. Phys. Lett. 81, 4637 (2002)

${ }^{5}$ E. A. Shaner, A. D. Grine, M. C. Wanke, M. Lee, J. L. Leno, and S. J. Allen, IEEE Photon. Technol. Lett. 18, 1925 (2006).

${ }^{6}$ G. C. Dyer, G. R. Aizin, J. L. Reno, E. A. Shaner, and S. J. Allen, IEEE J. Sel. Top. Quantum Electron. 17, 85 (2011).

${ }^{7}$ G. C. Dyer, S. Preu, G. R. Azin, J. Mikalopas, A. D. Grine, J. L. Reno, J. M. Hensley, N. Q. Vinh, A. C. Gossard, M. S. Sherwin, S. J. Allen, and E. A. Shaner, Appl. Phys. Lett. 100, 083506 (2012).

${ }^{8}$ H. Saxena, R. E. Peale, and W. R. Buchwald, J. Appl. Phys. 105, 113101 (2009).

${ }^{9}$ A. V. Muravjov, D. B. Veksler, V. V. Popov, O. V. Polischuk, N. Pala, X. Hu, R. Gaska, H. Saxena, R. E. Peale, and M. S. Shur, Appl. Phys. Lett. 96, 042105 (2010).

${ }^{10}$ N. Nader Esfahani, R. E. Peale, W. R. Buchwald, J. R. Hendrickson, and J. W. Cleary, Proc. SPIE 8512, 85120Y (2012).

${ }^{11}$ N. Nader Esfahani, R. E. Peale, W. R. Buchwald, J. R. Hendrickson, and J. W. Cleary, Proc. SPIE 8624, 86240Q (2012).

${ }^{12}$ J. W. Cleary, R. E. Peale, H. Saxena, and W. R. Buchwald, Proc. SPIE 8023, 80230X (2011)

${ }^{13}$ N. Nader Esfahani, R. E. Peale, J. W. Cleary, and W. R. Buchwald, Proc. SPIE 8023, 80230R (2011)

${ }^{14}$ A. A. Grinberg and M. S. Shur, J. Appl. Phys. 65, 2116 (1989).

${ }^{15}$ L. Zheng, W. L. Schaich, and A. H. MacDonald, Phys. Rev. B 41, 8493 (1990).

${ }^{16}$ R. E. Peale, H. Saxena, W. R. Buchwald, G. C. Dyer, and S. J. Allen, Jr., Proc SPIE 7311, 73110I (2009).

${ }^{17}$ L. D. Landau, E. M. Lifshitz, and L. P. Pitaevskii, Electrodynamics of Continuous Media, 2nd ed. (Elsevier, Butterworth Heinemann, Amsterdam, 1984). 